\title{
Hydroxyapatite Formation on/in Biodegradable Chitosan Hydrogels by an Alternate Soaking Process
}

\author{
Wanpen TACHABOONYAKIAT, Takeshi SERIZAWA, and Mitsuru AKASHI ${ }^{\dagger}$ \\ Department of Applied Chemistry and Chemical Engineering, Faculty of \\ Engineering, Kagoshima University, 1-21-40 Korimoto, Kagoshima 890-0065, Japan
}

(Received September 11, 2000; Accepted October 20, 2000)

\begin{abstract}
Assessment was made of the feasibility of forming hydroxyapatite on/in naturally occurring swollen biodegradable chitosan hydrogels through an alternate soaking process. This study focused on the effects of crosslinking and swelling on hydroxyapatite formation. Two chitosan solutions ( $1.75 \mathrm{wt} \%$ and $1.5 \mathrm{wt} \%)$ were prepared at various glutaraldehyde (GA) feed to obtain appropriate network structures. The amount of hydroxyapatite formed increased with swelling and decrease in crosslinking density. The hydrogel prepared from the $1.75 \mathrm{wt} \%$ chitosan solution showed more hydroxyapatite formed on/in the swollen chitosan hydrogel matrix than the $1.5 \mathrm{wt} \%$ chitosan solution hydrogel at the same GA feed and/or swelling. The swelling of the chitosan-hydroxyapatite composites was smaller than that of the chitosan hydrogel. The three-dimensional structure of the hydroxyapatite composite could be controlled by manipulating certain parameters of the starting chitosan hydrogel.
\end{abstract}

KEY WORDS Chitosan / Hydrogel / Hydroxyapatite / Alternate Soaking Process /

Biological and biomedical applications of polymeric materials have increased recently. As biomedical material, calcium phosphate is considered bioactive and to have good osteoconductive properties. ${ }^{1}$ The most widely occurring biological calcium phosphate is hydroxyapatite. Hydroxyapatite, the main inorganic component of bones and teeth, is one of the most biocompatible inorganic materials, with biomedical applications. Applications are limited as hard tissues. Several investigators have studied implant materials for replacement of the hard tissues, such as titanium and titanium alloy coated with apatite. $^{2-3}$ There is little research on soft implant materials for soft tissue adhesion. Therefore, several hydrogel-hydroxyapatite composites were prepared and evaluated as novel implantable materials that contact soft tissue.

Several techniques have been investigated to produce apatite crystal deposition on a substrate, such as thermal sprayed and plasma sprayed coating, ${ }^{4-7}$ precipitation, ${ }^{8}$ and soaking in simulated body fluid. ${ }^{9}$ From a historical point of view, the biomimetic process, the socalled "Kokubo Method" was first developed by Kokubo et al. ${ }^{10-11}$ We developed an alternate soaking process ${ }^{12}$ with great potential to form calcium phosphate on/in biomaterials over a short period of time. Many studies have investigated bone-like hydroxyapatite formation on/in various polymer materials. Taguchi et al. focused on hydroxyapatite formation on/in polyvinyl alcohol hydrogels ${ }^{13-15}$ and hydrophilic polymer grafted films ${ }^{16}$ using an alternate soaking process.

Chitosan, a naturally occurring polysaccharide obtained by deacetylation of chitin, is excellent for biomedical application ${ }^{17}$ because of its biodegradability and biocompatibility. Chitosan has the ability to swell in water into soft rubbery consistency, resembling living tissue. A swollen chitosan hydrogel, which acts as an electrically positive polymeric matrix, should thus induce hydroxyapatite formation on/in the chitosan net-

${ }^{\dagger}$ To whom all correspondence should be addressed. work by an alternate soaking process. Several bioactive molecules such as drugs and proteins can be covalently or electrostatically conjugated. To develop a bone mimic material, the preparation and characterization of a positively charged, chitosan hydrogel-hydroxyapatite composite was studied. The effects of swelling of the chitosan hydrogels on hydroxyapatite formation were studied using an alternate soaking process.

\section{EXPERIMENTAL}

\section{Materials}

Chitosan 500 with molecular weight of 1200000 (determined by high performance liquid chromatography (HPLC) using acetate buffer as the mobile phase and calibrated with a pullulan strandard) and disodium hydrogenphosphate $\left(\mathrm{Na}_{2} \mathrm{HPO}_{4}\right)$ were purchased from Wako Pure Chemical Industries, Ltd., Osaka, Japan. Tris(hydroxymethyl)aminomethane (Tris) was from Sigma Chemical Co., Steinheim, Germany. Glutaraldehyde (GA) and calcium chloride $\left(\mathrm{CaCl}_{2}\right)$ were supplied from Nacali Tesque Inc., Kyoto, Japan. All these chemicals were used without further purification.

\section{Chitosan Hydrogel Preparation}

Glutaraldehyde (GA) is an effective crosslinking reagent for producing a gel from an aqueous chitosan solution. ${ }^{18}$ Chitosan solutions of $1.5 \%$ and $1.75 \%$ by weight were prepared in $0.5 \%$ by volume acetic acid. A $120-240$ $\mu \mathrm{L}$ aliquot of a $25 \%$ glutaraldehyde aqueous solution was added to $15 \mathrm{~mL}$ chitosan solution as summarized in Table I. Chitosan solutions of $1.5 \%$ and $1.75 \%$ by weight seemed suitable concentrations in a preliminary study. Chitosan hydrogels cannot form under $1.5 \%$ by weight, even in the presence of high crosslinking concentration. If the chitosan concentration is over $1.75 \%$ by weight, a chitosan hydrogel will form very rapidly after adding the crosslinking agent without control over the three dimensional structure, thus making it difficult to prepare disk shaped hydrogels. The mixture was poured into a mold 
Table I. Comparison of swelling of chitosan hydrogel with that of chitosan-hydroxyapatite composite

\begin{tabular}{|c|c|c|c|c|}
\hline Chitosan concentration & $\frac{\text { GA feed }}{\mu \mathrm{L} \mathrm{mL}^{-1}}$ & $\begin{array}{l}\text { Avg. diameter of swollen } \\
\text { chitosan gel / mm }\end{array}$ & Swelling of chitosan gel & $\begin{array}{c}\text { Swelling of chitosan- } \\
\text { hydroxyapatite composite }\end{array}$ \\
\hline \multirow[t]{5}{*}{$1.5 \mathrm{wt} \%$} & 8 & 23 & 490 & 6 \\
\hline & 10 & 19 & 300 & 8 \\
\hline & 12 & 17 & 225 & 9 \\
\hline & 14 & 16 & 204 & 9 \\
\hline & 16 & 12 & 172 & 6 \\
\hline \multirow[t]{5}{*}{$1.75 \mathrm{wt} \%$} & 8 & 23 & 694 & 5 \\
\hline & 10 & 18 & 314 & 6 \\
\hline & 12 & 15 & 179 & 7 \\
\hline & 14 & 13 & 140 & 8 \\
\hline & 16 & 11 & 131 & 5 \\
\hline
\end{tabular}

which had a silicone rubber spacer of $1 \mathrm{~mm}$ thickness sandwiched between glass plates. The crosslinking reaction was allowed to proceed in the mold at room temperature for $24 \mathrm{~h}$. A clear, amber hydrogel was formed with high glutaraldehyde content. The chitosan hydrogel was punched out into $10 \mathrm{~mm}$ disks, and swollen in distilled water at room temperature for 2 days. The swollen chitosan hydrogels were kept in distilled water at room temperature until use.

Swelling of the chitosan hydrogel may be calculated from the following equation:

$$
S R=\frac{W-W_{0}}{W_{0}}
$$

where $S R$ is the swelling ratio, $W$ weight of the swollen chitosan hydrogels, and $W_{0}$ is the weight of the dry chitosan hydrogel.

\section{Hydroxyapatite Formation on/in the Swollen Chitosan Hydrogel}

The hydroxyapatite formation on/in the swollen chitosan hydrogel was achieved by an alternate soaking process according to our previous study, ${ }^{12}$ by alternate immersion into two aqueous solution containing calcium and phosphate ions as shown in Figure 1. The swollen chitosan hydrogel disk was first immersed in $10 \mathrm{~mL}$ $\mathrm{CaCl}_{2}(200 \mathrm{mM}) / \mathrm{Tris}-\mathrm{HCl}(\mathrm{pH} 7.4)$ aqueous solution at $37^{\circ} \mathrm{C}$ for $2 \mathrm{~h}$, followed by rinsing at $37^{\circ} \mathrm{C}$ with distilled water. After rinsing, the calcium-laden swollen chitosan hydrogel fulfilled was immersed in $\mathrm{Na}_{2} \mathrm{HPO}_{4}(120 \mathrm{mM})$ aqueous solution at $37^{\circ} \mathrm{C}$ for $2 \mathrm{~h}$ and washed by a similar process. This alternate soaking was repeated 5 times to allow the formation of hydroxyapatite on/in the hydrogels. The chitosan-hydroxyapatite composite was freezedried. \%hydroxyapatite is obtained as,

$$
\% \mathrm{HAp}=\frac{W_{\mathrm{ap}, \mathrm{d}}-W_{0}}{W_{0}} \times 100
$$

where \%HAp percentage of hydroxyapatite in the swollen chitosan hydrogel, $W_{\text {ap,d }}$ weight of dried chitosan-hydroxyapatite composites, and $W_{0}$, weight of chitosan hydrogel in a dry state.

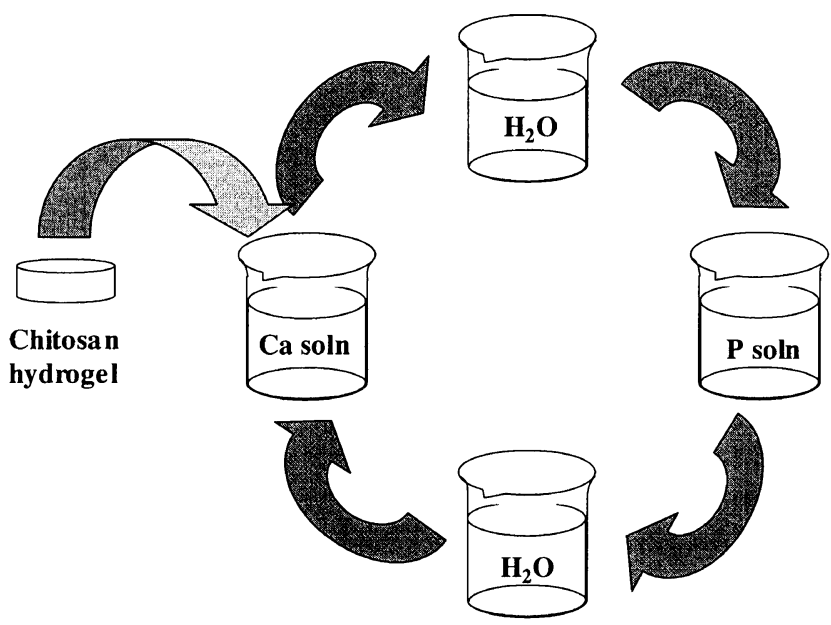

Figure 1. Experimental procedure for obtaining hydroxyapatite.

\section{Swelling of the Chitosan-Hydroxyapatite Composites}

Swelling of the chitosan-hydroxyapatite composites was studied in comparison to the chitosan hydrogels and was calculated as follows:

$$
S R_{\mathrm{ap}}=\frac{W_{\mathrm{ap}, \mathrm{w}}-W_{0}}{W_{0}}
$$

where $S R_{\text {ap }}$ is the swelling ratio of chitosan-hydroxyapatite composites, $W_{\mathrm{ap}, \mathrm{w}}$ wet weight of the chitosan-hydroxyapatite composite, and $W_{0}$, dry weight of the swollen chitosan hydrogel.

\section{$X$-Ray Diffraction Analysis}

X-Ray diffraction was measured by a RIGAKU Geigerflex 2013. Cu- $K_{\alpha}(\lambda=0.154 \mathrm{~nm})$ was used as the X-Ray source, and operated at $30 \mathrm{kV}, 15 \mathrm{~mA}$, and scan speed of $2^{\circ} \mathrm{min}^{-1}$ at $500 \mathrm{cps}$. The dried sample was placed on the glass slide specimen holder for examination at incident angles $2 \theta$ of $15-55^{\circ}$.

\section{RESULTS AND DISCUSSION}

The wet method is usable for mass production of small crystalline or non-crystalline hydroxyapatite powder. ${ }^{19}$ Hydroxyapatite was formed on/in a swollen chitosan hydrogel by our alternate soaking process. The reaction involves salt formation between calcium and phosphate 
ions. The chemical formula is as follows: ${ }^{19}$

$$
\begin{aligned}
10 \mathrm{CaCI}_{2}+6 \mathrm{Na}_{2} \mathrm{HPO}_{4}+2 \mathrm{H}_{2} \mathrm{O} \longrightarrow \\
\mathrm{Ca}_{10}\left(\mathrm{PO}_{4}\right)_{6}(\mathrm{OH})_{2}+12 \mathrm{NaCI}+8 \mathrm{HCI}
\end{aligned}
$$

\section{Chitosan Hydrogel Preparation}

Glutaraldehyde, an effective crosslinking agent for the functional amine group of chitosan, was added to the chitosan aqueous solution at various feeds to vary crosslinked content. ${ }^{20-22}$ A chitosan hydrogel disk with a $10 \mathrm{~mm}$ diameter and $1 \mathrm{~mm}$ thickness was prepared with $96 \%$ conversion, and swollen in distilled water for 2 days. The color of the hydrogels was yellow to brown, depending on the amount of glutaraldehyde. Water molecules slowly dispersed into the chitosan network, and Figure 2 shows the swelling of cationic chitosan hydrogels after reaching equilibrium. The hydrogels expanded more at lower glutaraldehyde feed. The hydrogel expanded by electric repulsion of remaining ammonium ions that can absorb several hundreds times their weight in water when measured against the original weight of the hydrogel. Figure 3 shows the degree of swelling of the chitosan hydrogels in distilled water plotted as a function of crosslinking agent feed. Swelling of the chitosan hydrogels decreased parabolically by increasing the GA feed. This may be due to the effects of remaining ammonium groups after crosslinking. There are many excess ammonium groups left over in lower GA feed, which may attract water molecules around them and thus swell the gel.

\section{Hydroxyapatite Formation on/in the Swollen Chitosan Hydrogels}

Positive charge of the chitosan was important in deciding the soaking extent of calcium and phosphate solutions. If the swollen, cationic, chitosan disk-shaped hydrogel is first immersed in anionic phosphate solution, phosphate ions surround the ammonium ions in the chitosan by ionic interaction and do not react with the calcium ions. The swollen chitosan hydrogels thus shrink. Hydroxyapatite forms in small amounts (data not shown here). In comparison to our previous study, ${ }^{14}$ the amount of hydroxyapatite formed on/in the poly(vinyl alcohol) (PVA) hydrogels (nonionic polymer) using the soaking order of $\mathrm{Ca}^{2+}-\mathrm{PO}_{4}{ }^{3-}$ was similar to that using the $\mathrm{PO}_{4}{ }^{3-}$ $\mathrm{Ca}^{2+}$ soaking order. Therefore, in this study, the swollen chitosan hydrogel disks were first immersed in calcium chloride solution. The calcium ions displace free water molecules, and repulse each other to give enough space to react with phosphate ions. The chitosan network gradually shrank and enclosed calcium ions inside. The hydrogel disks were washed with distilled water to get rid of excess calcium ions before soaking in disodium hydrogenphosphate solution. Initially, the hydroxyapatite formed only on the surface of the swollen chitosan hydrogels. The resulting hydroxyapatite deposits seemed to function as nucleating particles to induce calcium and phosphate ions to enter the swollen chitosan hydrogels for the next alternate cycle. The hydroxyapatite was completely dispersed throughout the swollen chitosan hydrogel after 5 cycles. Figure 4 shows a cross section and top surface of the chitosan-hydroxyapatite composite after 1 cycle and 5 cycles.

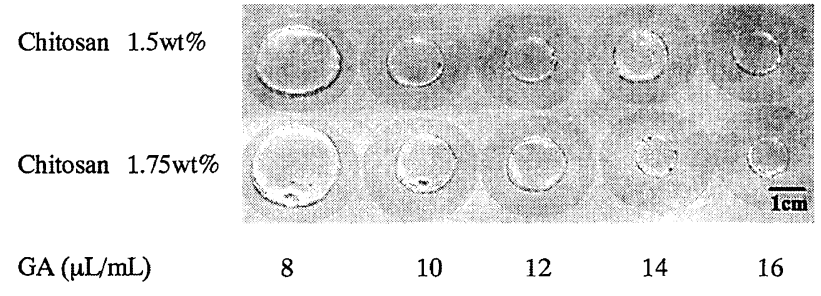

Figure 2. Appearance of swollen chitosan hydrogel at various crosslinking agent feeds in most expanded state.

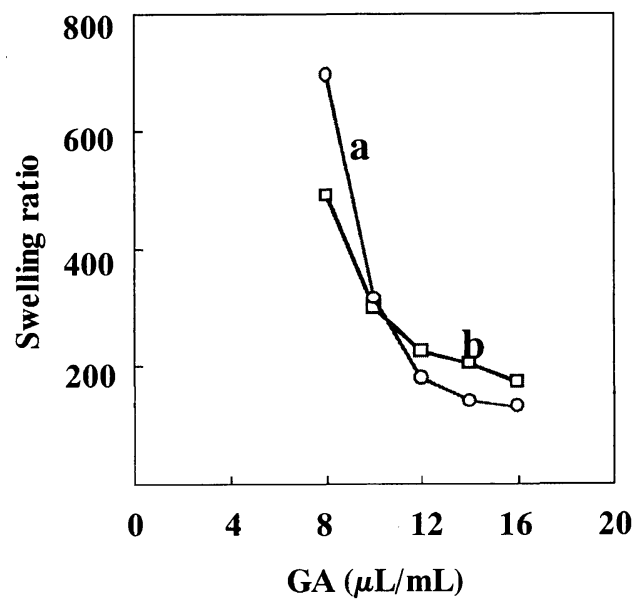

Figure 3. Relationship between glutaraldehyde feed and swelling of chitosan hydrogel, (a) 1.75 wt\%, (b) $1.5 \mathrm{wt} \%$.

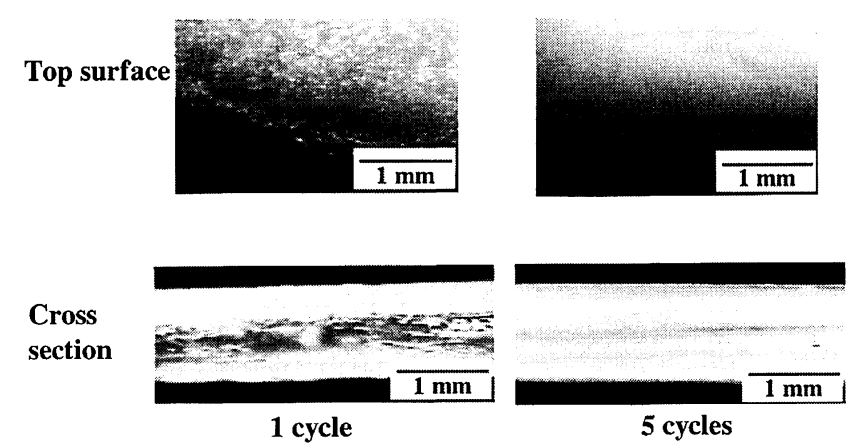

Figure 4. Microscopic observation of chitosan-hydroxyapatite composite after 1 and 5 cycles alternate soakings: Top surface and cross section pictures at magnification $\times 100$.

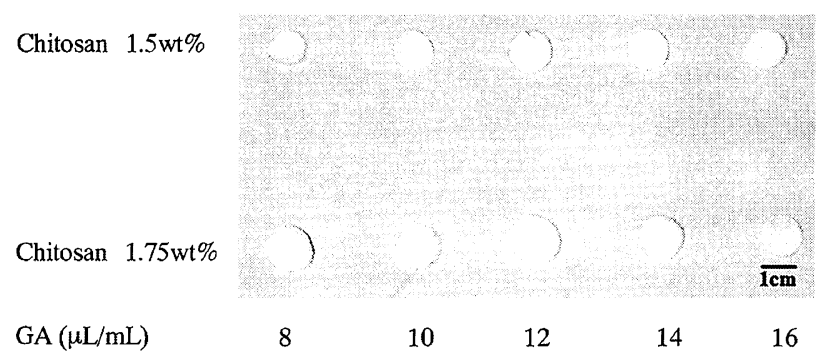

Figure 5. Appearance of chitosan-hydroxyapatite composite after 5 alternate soakings.

Figure 5 shows the appearance of the chitosan-hydroxyapatite composites after 5 calcifications. From the photographs, it is clear that an inorganic substance, pos- 
sibly the hydroxyapatite, has formed on/in the swollen chitosan hydrogels. The size of the chitosan-hydroxyapatite composite returned to approximately $7-9 \mathrm{~mm}$ in diameter, which is comparable to the chitosan hydrogels (10 $\mathrm{mm}$ diameter) before swelling in water. The condensation of swollen hydrogels may result from change in the ionic concentration and subsequent reduction in the osmotic swelling pressure inside the hydrogels. These results suggest that the three dimensional shape of the hydroxyapatite composites is controlled by the starting shape of the chitosan hydrogel. This method can be applied to bone-like materials in various shapes. The swelling of chitosan-hydroxyapatite composite is dramatically less than that of the chitosan hydrogels, as shown in Table I. The water adsorption capacity of the chitosan -hydroxyapatite composites is minimized by high \%hydroxyapatite formation, and this is a major advantage in implanting bone-like chitosan-hydroxyapatite composites into humans or animal bodies without changing their structure.

\section{Effect of the Swelling Ratio on the Quantity of Hydroxya- patite}

Figure 6 shows the amount of hydroxyapatite formed on/in the swollen hydrogels as a function of swelling of the hydrogel. The swollen hydrogel with higher swelling (i.e., a lower degree of cross-linking) had a larger \%hydroxyapatite deposited in the chitosan matrix, possibly because the swollen hydrogel can facilitate the diffusion of $\mathrm{Ca}^{2+}$ and $\mathrm{PO}_{4}{ }^{3-}$ ions into the chitosan network. The higher the chitosan concentration, the greater the amount of hydroxyapatite. The amount of hydroxyapatite with respect to swelling was evaluated to examine the equilibrium between hydroxyapatite formation and swelling. $1.75 \mathrm{wt} \%$ and $1.5 \mathrm{wt} \%$ swollen chitosan hydrogels showed plateaus in the amount of hydroxyapatite formed after swelling of around $200 \%$ and $300 \%$, respectively. There must be a critical chitosan concentration and cross-linking density for the most effective hydroxyapatite formation using this alternate soaking process. Overly high cross-linking density leads to hard and brittle hydrogels, resulting in a compact network which inhibits hydroxyapatite formation.

\section{$X$-Ray Diffraction Analysis}

The formation of hydroxyapatites on/in the swollen chitosan hydrogels was measured based on X-Ray diffraction. To evaluate the chitosan matrix related to hydroxyapatite formation, X-Ray diffraction of chitosan hydrogel and composites was examined as shown in Figure 7. Hydroxyapatite-appropriate peaks were observed at $26^{\circ}$ and $32^{\circ}$, and chitosan hydrogels at $12 \mu \mathrm{L} \mathrm{mL}{ }^{-1} \mathrm{GA}$ feed showed broad peaks at $22^{\circ}$. X-Rays of hydroxyapatite overlapped the chitosan peaks, and thus the hydroxyapatite forms over the surface of the swollen chitosan hydrogel. In the case of low GA feed hydrogel, i.e., high swelling for the chitosan hydrogel, $\mathrm{Ca}^{2+}$ and $\mathrm{PO}_{4}{ }^{3-}$ ions diffuse into the network easily and X-Ray peaks of hydroxyapatite are clearly observed. X-Rays of hydroxyapatite may be observed after 5 alternate soakings, which implies that hydroxyapatite forms within a short period of time in vitro at high quantities.

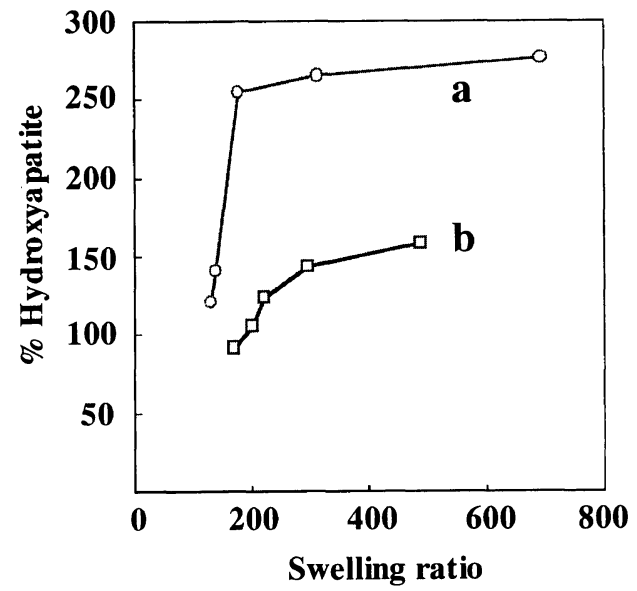

Figure 6. Effects of swelling $(S R)$ of chitosan hydrogels on amount of hydroxyapatite after 5 soakings, (a) $1.75 \mathrm{wt} \%$, (b) 1.5 wt\%.

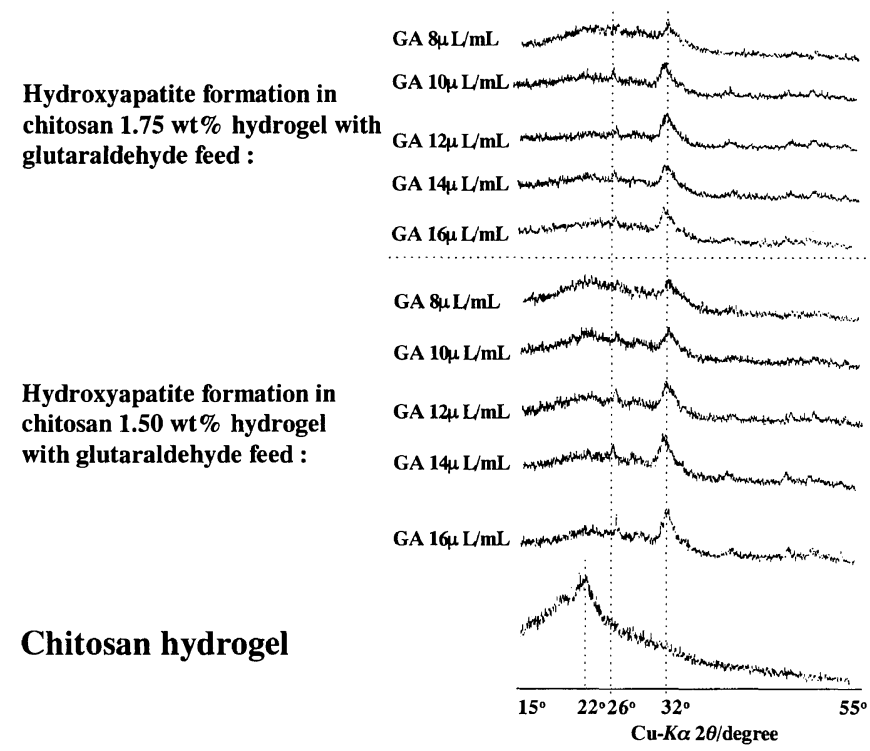

Figure 7. X-Ray diffraction of hydroxyapatite formed on/in the swollen chitosan hydrogels.

\section{CONCLUSION}

Chitosan was used to produce a cationic polymeric hydrogel for obtaining hydroxyapatite-organic polymer composite material. Soaking order was fixed to start with calcium solution, which was controlled by the cationic charge of the chitosan matrices. The hydroxyapatite formed on/in the hydrogels over a short period of time by alternately immersion in calcium chloride and disodium hydrogenphosphate solution at body temperature $\left(37^{\circ} \mathrm{C}\right)$. The three-dimensional shape of the chitosan -hydroxyapatite composite can be controlled by changing the shape of the starting chitosan hydrogels.

Acknowledgment. The authors thank the Minister of Education, Science, Sports, and Culture of Japan for a scholarship to W. Tachaboonyakiat and appreciate the assistance of Prof. A. Kishida, as critical advice throughout this research. 


\section{REFERENCES}

1. L. Cleries, J. M. Fernandez-Pradas, and J. L. Morenza, J. Biomed. Mater. Res., 49, 43 (2000).

2. A. P. Valagao, A. do Serro, A. C. Fernandes, and B. J. V. Saramago, J. Biomed. Mater. Res., 49, 345 (2000).

3. B. C. Yang, J. Weng, and X. D. Zhang, J. Biomed. Mater. Res., 47, 213 (1999).

4. H. Zeng and W. R. Lacefield, Biomaterials, 21, 23 (2000).

5. J. D. Haman, K. K. Chittur, D. E. Crawmer, and L. C. Lucas, J. Biomed. Mater. Res., 48, 856 (1999).

6. B. J. Story, A. V. Burgess, D. La, and W. R. Wagner, J. Biomed. Mater. Res., 48, 841 (1999).

7. Z. E. Erkmen, J. Biomed. Mater. Res., 48, 861 (1999).

8. H. Morgan, R. M. Wilson, J. C. Elliott, S. E. P. Dowker, and P. Anderson, Biomaterials, 21, 617 (2000).

9. I. Izquierdo-Barba, A. J. Salinas, and M. Vallet-Regi, J. Biomed. Mater. Res., 47, 243 (1999).

10. T. Kokubo, J. Non-Cryst. Solids, 120, 138 (1990)

11. G. J. Liu, F. Miyaji, T. Kokubo, H. Takadama, T. Nakamura, and A. Murakami, J. Mater. Sci.: Mater. Med., 9, 285 (1998).

12. T. Taguchi, A. Kishida, and M. Akashi, Chem. Lett., 1998,
711.

13. T. Taguchi, A. Kishida, and M. Akashi, J. Biomater. Sci., Polym. Ed., 10, 331 (1999).

14. T. Taguchi, A. Kishida, and M. Akashi, J. Biomater. Sci., Polym. Ed., 10, 795 (1999).

15. T. Taguchi, M. Shiraogawa, A. Kishida, and M. Akashi, J. Biomater. Sci., Polym. Ed., 10, 19 (1999).

16. T. Taguchi, Y. Muraoka, H. Matsuyama, A. Kishida, and M. Akashi, Biomaterials, 22, 53 (2001).

17. Q. Li, E. W. Grandmaison, and M. F. A. Goosen, in "Applications of Chitin and Chitosan", M. F. A. Goosen, Ed., Technomic Publishing Company, Inc., Lancaster, 1997, chapter 1.

18. R. A. A. Muzzarelli, in "Chitin", Pergamon Press, Oxford, 1997, pp 134-137.

19. H. Aoki, in "Medical Applications of Hydroxyapatite", Ishiyaku EuroAmerica, Inc., Tokyo, 1994, chapter 8, p 176.

20. L. Noble, A. I. Gray, L. Sadiq, and I. F. Uchegbu, Int. J. Pharm., 192, 173 (1999).

21. M. Kawase, N. Michibayashi, Y. Nakashima, N. Kurikawa, K. Yagi, and T. Mizoguchi, Biol. Pharm. Bull., 20, 708 (1997).

22. Y. Ohya, T. Takei, H. Kobayashi, and T. Ouchi, J. Microencapsulation, 10, 1 (1993). 